\title{
La violencia escolar desde la teoría del riesgo y el cambio cultural ${ }^{1}$
}

Mario Sandoval

Doctor en Sociología

\section{Resumen}

El presente artículo aborda el fenómeno de la violencia escolar desde la perspectiva de la teoría del riesgo en un contexto de profundo proceso de cambio cultural. Se trata de comprender el fenómeno de la violencia en los jóvenes desde una determinada perspectiva teórica, en un contexto determinado.

\section{Palabras clave}

Juventud / violencia / riesgo / escuela / educación

1 Este artículo es producto de la investigación FONDECYT N ${ }^{\circ}$ 1040694: "Figuras estructurales de la violencia escolar. Hacia una recuperación de la "subjetividad" educativa". 


\section{Abstract}

The present article approaches the phenomenon of students' violence from the perspective of the risk theory in a context of deep process of cultural change. The matter is to understand the phenomenon of violence in the young people from a determined theoretical perspective, in a certain context.

Key words

Youth / violence / risk / school / education 


\section{I.- Presentación:}

$\mathrm{E}$

1 presente artículo aborda el fenómeno de la violencia escolar desde la perspectiva de la teoría del riesgo en un contexto de profundo proceso de cambio cultural. Se trata de comprender el fenómeno de la violencia en los jóvenes desde una determinada perspectiva teórica, en un contexto determinado.

La violencia siempre ha sido un tema de análisis controvertido; por una parte, tenemos los hechos concretos que originan actos de violencia y, por otro,-simultáneamente- tenemos la impresión o percepción que la población -la opinión pública- tiene de esos hechos.

Lo primero que se advierte al analizar el fenómeno, es el desfase entre ambos elementos. Una cosa es la "temperatura" y otra (muy distinta a veces) es la "sensación térmica"; por lo general, la población tiene una sensación de inseguridad y temor que no guarda relación con los hechos reales y concretos que originan esa sensación.

Cuando nos encontramos frente a este fenómeno, necesariamente tenemos que hacer alusión al rol que juegan los mass-media en este proceso. Todo parece indicar que, cuando un hecho de violencia particular y específico, acaecido al interior de un establecimiento escolar es difundido profusamente por la televisión, el espectador tiende a generalizar y a "crearse" una imagen de una violencia generalizada que no guarda relación con la intensidad, 
proporción y frecuencia de los hechos violentos concretos ocurridos.

Lo anterior va generando una imagen de un joven violento, al cual hay que temer porque puede reaccionar en cualquier momento $y$, como Goffman lo dijera hace un par de décadas, del prejuicio al estigma hay un paso.

Producto del impacto televisivo en la audiencia acrítica, se va generando un etiquetaje social de los jóvenes chilenos y en particular de cierto tipo de jóvenes, especialmente los que provienen de sectores sociales subalternos que acceden a la educación municipalizada.

En el caso francés, desde los años '80, las periferias urbanas parecen haber llegado a ser el teatro de una exclusión creciente. Pareciera ser que las llamadas "cités de banlieus" ("poblaciones" en nuestro caso chileno) están en franco proceso de ghettizisación. La incivilidad y la delincuencia de los jóvenes habitantes de esos espacios urbanos cuestionan la sociabilidad más elemental.

Por su parte, las brutalidades, la degradación y las transgresiones a sus derechos más elementales no parecen detenerse, generando una dialéctica de causa-efecto que también es posible observar en los jóvenes chilenos marginados y excluidos de los beneficios del sistema económico. Sin embargo, la violencia juvenil popular (y no popular) no tiene las dimensiones dramáticas de otros países; el problema existe, pero no en una dimensión que cuestione el orden social imperante, es decir, no estamos ante la presencia de una violencia revolucionaria generalizada que cuestiona el orden imperante y propone otro tipo de orden social, sino, más bien, ante la presencia de actos menores, esporádicos, que no trascienden más allá del espacio institucional donde fueron generados. 


\section{II.- Acerca del Riesgo:}

Los orígenes del término riesgo parecen perderse en el tiempo, desconociéndose la existencia de estudios que den cuenta de su uso a través de la historia (Luhmann, 1992). Recién se tuvieron noticias del término a partir de la aparición de la imprenta, aunque su utilización aún era rara y principalmente aplicada al contexto de la navegación marítima. Los seguros marítimos constituyen un caso temprano de control de riesgo planificado.

Para Giddens (1994), la noción de riesgo aparece en el pensamiento europeo alrededor del siglo XIX con la palabra inglesa que se solía deletrear en su versión francesa como risque. Durante algún tiempo, la grafía francesa continuó utilizándose junto con la nueva palabra anglicanizada risk, que comenzó a emplearse en el campo de los seguros.

Douglas (1985), reconocida por sus trabajos antropológicos sobre riesgo y cultura, ha observado que la connotación de la palabra "riesgo" ha ido cambiando a través del tiempo. Para la autora, la noción de riesgo fue introducida en el siglo XVII en el contexto del juego, indicando la probabilidad de que un hecho ocurriera, combinada con la magnitud de pérdidas y ganancias. Todavía el concepto tenía un valor neutral. Hoy, la noción de riesgo tiende a estar asociada a resultados negativos, en especial en un uso profesional o técnico del término. La Royal Society, citada por Douglas (1982) definió riesgo como "la probabilidad de que ocurra un hecho particular adverso durante un periodo de tiempo dado".

En el uso científico del término, argumenta Hansson (1989), riesgo es considerado como un concepto unidimensional que hace referencia a un valor numérico de probabilidad, mientras que, en el uso popular, tiene muchas y más variadas acepciones. 


\section{III.- El riesgo desde la sociología}

En primer lugar, abordaremos la existencia de visiones más tradicionales acerca de la temática del riesgo; éstas pueden ser definidas como Técnico-Científicas (cf. Farrás, Bosch y Torrente 2001: 2), preponderantes en los estudios realizados por la Economía, Ingeniería, Epidemiología, Psicología e incluso por la Ciencia Jurídica. Bajo estas premisas, el riesgo se define esencialmente mediante expresiones referentes a probabilidades y resultados adversos, normalmente como secuela de una determinación meditada o reflexionada.

El objetivo de este enfoque se centra principalmente en la identificación de riesgos, determinación de sus factores causales, elaboración de modelos predictivos que enlacen diversos riesgos y en el examen de la percepción, análisis y toma de decisiones con relación a las diversas situaciones de riesgo, adoptando una perspectiva racionalista y realista en la que prevalece la visión del especialista, quien mide los riesgos y determina la forma de proceder ante los mismos (cf. Farrás, Bosch y Torrente 2001: 2).

Por su parte, en los enfoques pertinentes a la Sociología, el tema del riesgo no se plantea como una cuestión que aborda lo meramente técnico y adopta características de problemática social. Las diversas miradas sociológicas, en general, critican la concepción racionalista del riesgo y enfatizan que existe una construcción social del mismo. (cf. Farrás, Bosch y Torrente 2001: 2).

En estos términos, el "riesgo es dinámico y cambiante y su expresión más acotada se capta en unidades territoriales y sociales de pequeña escala (...). El riesgo es siempre producto de las acciones conscientes o inconscientes de actores sociales, organizaciones, instituciones o individuos" (Argüello y Lavell 2001: 3). 
Desde el punto de vista sociológico, a diferencia de las miradas técnicas y sicológicas, lo que interesa más bien son los factores que hacen que determinados puntos de vista respecto a los riesgos resulten dominantes en grupos sociales determinados, entre ellos los jóvenes, así como también el motivo de que se produzcan al respecto polarizaciones y controversias. Las opiniones y actitudes de los sujetos frente al riesgo son factibles de usar para describir la situación anterior. "En este sentido, la correlación de la distribución de las opiniones con factores socioculturales sirve de base para realizar análisis de potenciales diferencias respecto a estructuras sociales dadas", (López 2001: 7).

Desde la perspectiva sociológica, los diferentes grupos sociales elaboran variadas concepciones sobre la gravedad y aceptabilidad de distintas situaciones de riesgo, así como también acerca de las respuestas precedentes ante cada una de estas condiciones. Dichos patrones se rigen por los procedimientos acostumbrados de socialización y de factores económicos, políticos y culturales y no tanto del cálculo de probabilidades. En consecuencia, es posible afirmar que habrá una diferenciación entre la percepción del riego y el riesgo objetivo, lo cual es preocupante en el caso de la violencia en establecimientos escolares, dado que muchas veces un acto de violencia tiene su origen en un juego brusco donde no se midió el riesgo de la acción gatillante (cf. Farrás, Bosch y Torrente 2001: 2).

Los riesgos emergen, entonces, como derivaciones de procedimientos socioculturales que sirven a determinadas funciones sociales y políticas, siendo objeto de estudio el origen y sus consecuencias sociales, su uso simbólico, la forma en que se adjudican culpas y responsabilidades, el papel de los expertos y del conocimiento en su gestión, su reparto desigual, los conflictos asociados o su relación con los procesos de modernización y globalización. 
Recalcar la dimensión social y cultural del riesgo evita caer en etnocentrismos que nos llevan a tildar de arriesgadas conductas y actitudes de personas que no comparten el conocimiento o la estructura de valores y símbolos asociados a un determinado riesgo. $\mathrm{Al}$ respecto, el caso de la violencia intra-escolar es particularmente interesante, pues está asociado, consciente o inconscientemente al juego de la vida y la muerte.

Aunque parezca exagerada la afirmación anterior, es sabido que algunas acciones juveniles que comienzan como simples juegos entre muchachos terminan en un centro hospitalario o en el cementerio.

Con todo, como bien expresa el sociólogo Josep Espluga (2001: 13), con respecto al esfuerzo por dar un sustento global que legitime esta orientación, "hay que señalar que la incorporación de las dimensiones sociales al análisis empírico de los riesgos tecnológicos y ambientales es un paso importante para caminar en esta dirección".

Desde la particular óptica de la disciplina sociológica, se supone que el riesgo es un estado del individuo ante el peligro y se evita enfocarlo exclusivamente en términos de probabilidad de un suceso y sus consecuencias. Es más, Ronald Perry y Miguel Montiel (1996: 5) sostienen que hay que concebir el riesgo en el contexto de sus consecuencias para la vida de los individuos $y$, siguiendo a Anthony Wallace, señalan que hay que aproximarse al tema del riesgo considerando la noción de "desintegración total", vale decir, la aprensión de los seres humanos ante la capacidad de ciertos eventos naturales o societales de interrumpir instantáneamente el curso normal de sus vidas. En el caso de una conducta social violenta, por ejemplo, es fácilmente apreciable la gama de consecuencias asociadas al deterioro del lazo social y, como última instancia, la muerte prematura de los protagonistas de las acciones violentas. 
Al respecto, se establece que pueden considerarse fenómenos perturbadores de origen natural (geológicos e hidrometereológicos) y de origen humano (químicos, sanitarios y socio-organizativos). Los fenómenos perturbadores socio-organizativos tienen su matriz en las concentraciones humanas y en el funcionamiento de algún sistema de subsistencia que proporciona servicios básicos, siendo los principales ejemplos las marchas y concentraciones masivas, delitos y accidentes terrestres y aéreos.

En efecto, el hombre, a lo largo de su evolución, ha ido disminuyendo su dependencia del medio natural pero la ha aumentado con respecto a determinadas formas materiales dadas por él mismo, generando un ambiente socializado llamado "medio sociocultural-técnico", relacionado con el uso de la técnica y la tecnología, la cual le ha posibilitado adquirir cierto status con el que establece estructuras u organizaciones de carácter económico, social, político y cultural más o menos homogéneas y que se definen como sociedades.

Esta capacidad para desarrollar técnicas que permitan simplificar y mejorar el modo de vida en el espacio terrestre, ha traído, al mismo tiempo, la creación de un ambiente mucho más complejo, haciéndose necesaria la acción de pautas de conducta que permitan seguir manteniendo este espacio construido. Siguiendo las ideas de Chinoy, estas regularidades dentro de las sociedades reflejan la presencia de cultura y conocimiento de lo que supuestamente es controlable.

Pese a ello, al manifestarse el no seguimiento de las reglas establecidas, falta de habilidad o carencia de conocimiento de los actores sociales, imprudencia, temeridad, ignorancia, etc., se pierde el equilibro obtenido y, como consecuencia, algunos eventos que, dada su magnitud, frecuencia $u$ otro vocablo que lo defina como ajeno al orden social establecido, son declarados como problemas sociales. 
En definitiva, el sociológico es un acercamiento distinto a la noción de riesgo. Por ello, nos ha parecido necesario incorporarlo en el análisis de un fenómeno de la violencia escolar que puede llegar a ser analizado sólo desde el punto de vista psicológico.

Desde el punto de vista sociológico, el riesgo es definido subjetivamente por los sujetos afectados. Por tanto, existe un elemento implícito de cognición y percepción individual. Sin embargo, es perfectamente posible obtener cierta consistencia entre las diversas definiciones de los individuos a un grado tal que las normas y estilos de vida, y los efectos de un suceso, son similares. Por otro lado, el riesgo no es entendido únicamente en términos de daños a la propiedad, sino también de la discontinuidad generada en el curso de la vida cotidiana. En este sentido, el riesgo es visto tanto desde la perspectiva del bienestar individual como de la seguridad de familiares, amigos, compañeros de curso y comunidades. Es preciso entonces, que, para entender el riesgo, los científicos sociales integren los conceptos de daños a la propiedad y de amenazas a la vida.

El riesgo, como eje interpretativo de la sociedad contemporánea y de las nuevas formas de vida social de las última décadas, aparece en el ámbito de la teoría sociológica como resultado de la deliberación acerca de los límites que habría alcanzado el modelo histórico de la modernidad, fundado en la sociedad industrial y que Giddens (1990: 15) define como "aquellos modos de vida u organización social que surgieron en Europa desde alrededor del siglo XVII en adelante y cuya influencia, posteriormente, los ha convertido en más o menos mundiales".

En cuanto a Niklas Luhmann, el riesgo y la actitud de los individuos o grupos ante el mismo aparecen como ejes centrales de la modernidad, siendo la valoración y aceptación del riesgo no una cuestión síquica sino fundamentalmente social. La conducta individual ante el riesgo 
implica un ajuste a las expectativas socialmente mantenidas por los grupos de referencia relevantes o bien es el resultado consecuente de procesos de socialización específicos. Junto al cálculo, percepción, valoración y aceptación del riesgo, se suman los problemas de saber quién o qué decide si un riesgo debe ser considerado, estando el problema de la selección de riesgos orientado a dilucidar la posibilidad de que los factores sociales puedan dirigir este proceso de selección (cf. Pucci 1999:8).

Para Luhmann, el riesgo va más allá de la elección racional, sino que constituye como un problema social construido comunicacionalmente e históricamente diferenciado. El riesgo supone muchos factores asociados que afectan negativamente los resultados de las acciones, razón por la que es imposible su cálculo racional. Lo importante es que sea evitable tomando una adecuada decisión presente en cuanto a lo que pasará en el futuro. No obstante, es necesario tener presente que la seguridad jamás será completa dado que no existe conducta libre de riesgo, se tomen o no decisiones, (cf. Torres y De la Puente 2001 ).

En estos términos, no existiendo decisiones libres de riesgo, es poco viable que la investigación y la generación de conocimiento provoque el tránsito del riesgo a la seguridad. Para Luhmann, la experiencia demuestra todo lo contrario pues, mientras más se sabe, más se conforma una conciencia de riesgo, vale decir, mientras más racional y complejo sea el cálculo del riesgo, más aspectos se verán revelados y, con ellos, mayor incertidumbre y riesgo en el futuro. (cf. Medina 1985: 6).

Por su parte, el enfoque de la "Sociedad del Riesgo" (Ulrich Beck y Anthony Giddens) enfatizan los factores estructurales y macro de las sociedades modernas, señalando que el desarrollo científico y tecnológico implica una inseguridad cada vez mayor, indiscriminada y global, por 
lo cual el riesgo es más difícil de calcular, gestionar y evitar. Paradójicamente, las instituciones núcleo de la modernidad (Gobierno, Industria y Ciencia) son las que más riesgos generan. A su vez, las sociedades avanzadas demandan más seguridad y calidad de vida. Todo ello acontece en el marco de una individualización creciente de la sociedad en la cual las personas se ven como las indicadas para decidir sobre su propia seguridad (cf. Farrás, Bosch y Torrente 2OO1: 3).

Beck y Giddens comparten muchos puntos en común en cuanto al análisis del riesgo, principalmente al entenderlo como un componente de una segunda etapa de la modernidad marcada por la radicalización y universalización de sus características esenciales y por su negación (rechazando de paso los planteamientos referentes a la postmodernidad). La diferencia fundamental entre Beck y Giddens pasa por el hecho de que, mientras el primero se centra preferentemente en los problemas institucionales planteados por la modernidad reflexiva, siendo por tanto su enfoque más macro, el segundo se sitúa más en los conflictos de la identidad individual y el desarrollo del sujeto reflexivo, pudiendo calificarse su visión como más micro. Nugent afirma al respecto que "asi pues, el sujeto reflexivo de Giddens y la modernidad reflexiva de Beck se enfrentan a las nuevas formas de riesgo de la sociedad contemporánea" (Nugent 2001: 3).

Beck postula el concepto de "Sociedad del Riesgo" en alusión a un "estadio de la sociedad moderna en el que la producción de riesgos políticos, ecológicos e individuales está cada vez más fuera del control de las instituciones encargadas de garantizar la seguridad de la sociedad" (Espluga 2001: 9).

Beck define las características de las sociedades del riesgo ${ }^{2}$ centrándose en el hecho de que la ruptura profunda entre las sociedades industriales o modernas y las sociedades de riesgo o de la modernidad reflexiva, pasa por la

2 Véase al respecto BECK 2002 
sustitución del orden, la jerarquía y la autoridad que caracterizan a la primera, por la incertidumbre y la ambivalencia propios de la sociedad del riesgo, la cual no es sino producto del triunfo de la racionalidad y de la modernización que autodestruye sus propios fundamentos en su desarrollo.

Para Beck, la modernidad simple sitúa el cambio social en la racionalidad teleológica. En cambio, la modernidad reflexiva lo ubica en los efectos colaterales, no deseados e incontrolados, que no se ven ni se reflejan, pero que se externalizan mediante la acumulación de hechos latentes cuya interrelación provoca ruptura estructural, sustituyéndose los modelos de racionalización y modernización lineal por conceptos tales como auto-transformación, auto-amenaza y auto-destrucción, proceso que implica la crisis de la racionalidad y el retorno a la incertidumbre, generándose nuevas contradicciones y problemas a nivel societal, reflejados, por ejemplo, en que, a nivel de estructura social, se contrapongan las teorías de los grandes grupos a las teorías de la individualización (cf Pucci 1999:5).

\section{IV.-Acerca de la hipótesis de la Mutación Cultural}

La hipótesis de fondo que caracteriza el contexto en el cual se verifican las acciones de riesgo de los jóvenes y que, eventualmente derivan en acciones violentas, pertenece a Guy Bajoit y Abraham Franssen y señala que: "desde hace 20 ó 30 años, una mutación cultural está en curso"'3, es decir, estaríamos viviendo el paso "de un modelo cultural basado en la razón social a otro fundado en la autorrealización autónoma"4, y más aún, "la reducción de la credibilidad que afecta al modelo

3 Guy Bajoit et Abraham Franssen. Les Jeunes dans la Compétition Culturelle, Sociologie d'aujoud'hui, PUF, 1995. Pág. 185

4 G. Bajoit et al. Op. Cit. Pág. 186 
de la razón social y el aumento de la credibilidad que se vincula al modelo de la autorrealización autónoma serían, al final, un proceso irreversible en la medida en que éste sería alentado por todos, incluso por aquellos que aparentemente se esfuercen por resistirlo" ${ }^{\prime \prime}$.

El telón de fondo del cual parten los autores señalados es la idea de que hoy en día estaríamos viviendo un período de mutación cultural y que esta mutación dificulta que los jóvenes encuentren sentido a las cosas que hacen y -como consecuencia- desarrollan conductas contradictorias, contrapuestas, muchas veces incomprensibles para el mundo adulto. En un mundo que les exige cada vez más, se va produciendo un desfase entre las expectativas de éxito y los límites o los obstáculos que ellos perciben en el logro de esos objetivos. Ese desfase son las tensiones existenciales.

El problema del sujeto es un problema antiguo en la humanidad y, en cada época y lugar, el hombre busca ser sujeto, apelando al modelo cultural reinante, traducido en valores superiores o supremos (Dios, La Patria, La Naturaleza, etc.).

De esta manera, "el individuo es siempre sujeto, cualquiera sea el modelo cultural en el que viva, pero -de un modelo al otro- los principios de sentido a los cuales se apela para justificar su derecho a ser, son diferentes" $"$.

En un contexto cultural inestable, en mutación, el primer fenómeno que se produce es que las expectativas de los otros dejan de ser homogéneas y pasan a ser más o menos incoherentes; la socialización deviene paradojal. Los valores del antiguo modelo pierden su legitimidad progresivamente. Por lo tanto, la vía conformista ya no tiene sentido, aumentando cada día la cantidad de personas que buscan

5 G. Bajoit et al. Op. Cit Pág. 186

6 G. Bajoit et. al. Op. Cit. Pág. 180 
ser sujetos por la vía contestaria o marginal, asumiendo conductas de riesgo, algunas de ellas violentas.

Por otra parte, los individuos escapan a las formas instituidas de contestación y marginalidad, intentando fundar sus proyectos personales en principios culturales nuevos. El ser sujeto por la vía realista (del conformismo y de la movilidad) ahora toma la forma de una reafirmación de valores del pasado. Es así como cada vez más individuos forman parte de una situación de aculturación: ellos no pueden adherir al antiguo modelo cultural porque lo encuentran indeseable e impracticable, pero -a la vez- no pueden adherir al nuevo, dado que su legitimidad aún no está asegurada. Por lo tanto, se ven obligados a tratar de conciliar los dos modelos en sus prácticas cotidianas.

A partir de lo anterior, Bajoit et. al., plantean como hipótesis "que estaríamos pasando de un modelo cultural basado en la razón social (es legítimo aquello que es útil a la colectividad, es decir, contribuye a su progreso y obedece a su razón) a otro fundado en la autorrealización au tónoma (es legítimo aquello que el individuo juzga bueno para su desarrollo personal) en la medida que eso no impide a nadie hacer lo mismo"7.

\section{V.- Acerca de las lógicas de acción juveniles}

Frente a los planteamientos anteriores, quisiéramos evaluar dos interpretaciones posibles. Por una parte, la propuesta de la "sociología de la experiencia" de Francois Dubet y, por otra, la de la mutación del modelo cultural de Guy, Bajoit y Abraham Franssen.

$7 \quad$ Bajoit et. al. Op. Cit. Pág. 181 
Según F. Dubet, no estamos en presencia de "un" eje articulador que define " $e l$ " conflicto central que posiciona a los actores colectivos en función de "intereses comunes" y que actúan bajo "una" lógica única. Lo que se advierte en las conductas juveniles es, más bien, una separación entre la subjetividad individual y la objetividad del sistema, fragmentado en múltiples Lógicas de Acción.

Desde esta óptica, F. Dubet nos proporciona un concepto clave para comprender las conductas de los jóvenes. Este es el concepto de "experiencia", entendida como una "noción que designa las conductas individuales y colectivas dominadas por la heterogeneidad de sus principios constitutivos y por la actividad de los individuos que deben construir el sentido de sus prácticas en el seno mismo de esta heterogeneidad"8.

Actualmente, estamos ante la presencia de una diversidad de jóvenes que se mueven en espacios cotidianos distintos: unos, caracterizados por la pobreza, la marginación y la exclusión; otros, por la comodidad, el lujo y la abundancia En ese contexto, desarrollan ciertas lógicas de acción que les permiten sobrevivir, adaptarse, integrarse parcial y simbólicamente o conformarse a la exclusión forzada.

Coincidimos con Dubet en el sentido de que, al observar las conductas de esta nuevas generaciones, no es posible reducirlas a un rol determinado ni tampoco a la persecución de determinadas estrategias de interés, sino que -en sus conductas- se plasman tres características esenciales:

a) La primera se refiere a la heterogeneidad de los principios culturales y sociales que organizan sus conductas. Todo pasa como si ellos adoptaran simultáneamente muchos puntos de vista a la vez, como si su identidad estuviera configurada de movimientos identificatorios sucesivos. Es por eso que las tipologías deben servir como guías de

8 François Dubet, Sociologie de l'experience. La couleur des idées. Seuil. Paris. Octubre 1994. 
referencias, no como "etiquetas sociales"; hacerlo sería un error y no permitiría comprender y aprehender el fenómeno en toda su complejidad.

A partir de las definiciones de roles, status o pautas culturales rígidas, estables y preestablecidas no se puede comprender el comportamiento de los jóvenes. Ellos no constituyen una masa que cumple un programa determinado. Las nuevas generaciones juveniles no construyen una unidad a partir de un vacío social. Ellos no son parte de un guión en blanco que van "improvisando". Por el contrario, su espacio social está lleno de contenidos diversos que se entrecruzan, dando origen a una multiplicidad de Lógicas de Acción. En este sentido, sus identidades no son un "ser" dado, apriori, es un "trabajo", un proceso de construcción siempre dinámico.

b) La segunda característica de la conducta de los jóvenes es la distancia subjetiva que ellos mantienen con el sistema.

c) La tercera característica a la que se refiere Dubet es que la construcción de la experiencia colectiva recoloca la noción de alienación en el corazón del análisis sociológico.

El análisis nos parece válido y certero en lo que se refiere a la constitución de nuevos movimientos sociales, es decir, si desaparece la imagen clásica de "la sociedad", los nuevos movimientos sociales que se constituyen y los antiguos que aún subsisten no pueden apelar a la combinatoria de intereses colectivos, de utopías compartidas en función de proyectos globales que representen los intereses de "la clase", del "pueblo".

Al respecto, la tipología propuesta por Dubet se encuentra sobrepasada, caduca. No tiene sentido pensar y 
pretender comprender el complejo accionar de los jóvenes actuales si se les analiza sólo en el cruce de sus acciones colectivas: populismo, defensa comunitaria, reivindicación y ruptura revolucionaria. La tendencia juvenil predominante es a alejarse de las acciones colectivas y a estructurar los lazos sociales de manera diferente a la forma como se hiciera en las décadas pasadas.

Por su lado, según G. Bajoit, estaríamos viviendo un tiempo de mutación cultural. Los parámetros que le brindaron las certezas a las generaciones pasadas están siendo sobrepasados por un nuevo modelo que tiene como eje de articulación la "autorrealización autónoma".

Lo interesante de la propuesta de Bajoit es que le otorga un rol protagónico al sujeto mismo en el proceso de cambio. No son las estructuras que cambian y como acto reflejo los individuos cambian con ella, sino que -más bien- es un proceso conjunto que se retroalimenta incesantemente.

Las acciones que realizan los jóvenes contemporáneos son acciones cargadas de sentido, en un contexto que les proporciona un conjunto de sentidos culturales vehiculizados por representaciones, normas, valores e ideologías, las que, en su conjunto, configuran el modelo cultural que progresivamente se impone y lucha en su irrupción en la escena nacional con otro conjunto de representaciones, normas, valores e ideologías que constituyen otro modelo que subsiste, pero que pierde vigencia día a día.

De esta manera, el sujeto construye las estructuras de sentido que forjan sus expectativas en los distintos campos en los cuales se desenvuelve cotidianamente. Sin embargo, en el desarrollo de sus conductas, el " $y o$ " se encuentra con los otros, con las instituciones, con la legalidad vigente, con el "Estado de Derecho" y, en sus intercambios cotidianos, el "sujeto-actor" se enfrenta a un conjunto de presiones sociales y materiales que configuran una estructura de 
control que genera los límites que le impiden lograr sus expectativas.

La relación entre las expectativas y los límites da origen a incoherencias y contradicciones en cada ser humano, siendo la fuente de la tensión existencial que cada uno debe sobrellevar. El resultado es una sensación de malestar existencial que busca resolverse de alguna manera. La tensión entre "querer ser" y "deber ser" está siempre presente y, para gestionar esa tensión, el sujeto se defiende, se adapta, se reconstruye o salva su identidad personal, es decir, desarrolla diversas lógicas de acción, entendidas éstas como el eje central sobre el cual las personas articulan su conducta, lo que da origen a ciertas tendencias.

Como muy bien lo señala Bajoit, el vivir el tránsito de un modelo a otro, genera confusión, incertidumbre, ambiguedades, zonas poco claras donde el comportamiento humano se fragmenta en una pluralidad de lógicas de acción, adhiriendo, en algunos casos, al modelo cultural antiguo, viviendo de lleno el proceso de transición en otros $\mathrm{y}$, finalmente, en una tercera posibilidad, adhiriendo con claridad a los valores del nuevo modelo cultural en proceso de instalación.

\section{VI.-Conclusión:}

En general, en el mundo juvenil, quien transgrede la norma se siente importante y admirado por sus amigos, resultando estimulante el acto mismo de la transgresión; al respecto, todo parece indicar que es la relación "jovennorma" es uno de los espacios de mayor cambio cultural contemporáneo.

Una buena cantidad de jóvenes en los espacios escolares se presentan como transgresores, particularmente 
cuando se enfrentan a un espacio tan normativo como lo es el espacio escolar y el acto mismo resulta estimulante. Los jóvenes saben que esos actos serán reprobados por los adultos y eso le añade un poco de emoción. Lo que importa es la aprobación de los amigos; ésa es la recompensa y merece la pena asumir el riesgo del castigo.

Los casos leves de vandalismo y violencia forman parte del desarrollo normal de niños y jóvenes, provienen de su necesidad de sentirse independientes, rebeldes, o parte de un grupo; el de sus pares. Los sentimientos que impulsan estos actos son universales. Los jóvenes buscan identificarse como individuos y reafirmarse como miembros de un grupo. En otras ocasiones, buscan desquitarse de acciones que consideran injustas, protagonizadas por las figuras de autoridad: padres, profesores, policía. Una de las vividas como más injusta es la que convierte al joven en "invisible", todo lo que él o ella interpretan como que no se les tiene en cuenta o no se les reconocen sus logros.

Muchos niños y jóvenes que crecen en ambientes en los que sienten que no valen mucho, y pueden (por la excesiva tolerancia familiar) hacer cualquier cosa que le pida su grupo. La necesidad de aceptación por el grupo puede inducir a un comportamiento antisocial, especialmente en la adolescencia: en medio de la desorientación, sentirse parte del grupo (que, a veces, es lo único que eligen) es lo más importante.

Los actos agresivos son la punta del iceberg; el problema casi siempre es previo. A veces, los niños y jóvenes emiten "sus señales" con gran intensidad (un robo, una pelea con heridos, una agresión a los padres, a un compañero o profesor) y surge el problema, ya ineludible. El mensaje requiere respuestas.

F. Dubet asegura que "la violencia es gratuita", además de agregar que la rabia y el placer le son consustanciales. La imprevisibilidad y la gratuidad aparente de los actos 
violentos, el desarrollo de la delincuencia y el tráfico de drogas ponen en peligro la seguridad y los bienes públicos; a partir de aquello, surge un sentimiento de inseguridad, el cual ha sido ampliamente demostrado en los estudios de la Fundación Paz Ciudadana. Este sentimiento de inseguridad cuestiona un concepto central en la sociología contemporánea; nos referimos al concepto de "lazo social".

El debilitamiento del lazo social repercute en la disminución de la cohesión social; desde esta perspectiva, los jóvenes que protagonizan actos de violencia al interior de los espacios escolares (que son los menos) están buscando un protagonismo social negado extramuros, emergiendo como una figura simbólica del cuestionamiento del orden social establecido en el Chile actual: si estos actos de violencia no son controlados o bien canalizados, se corre el riesgo de debilitar, descomponer y desagregar el lazo social que cohesiona a la sociedad chilena.

El tema de la relación de los seres humanos entre sí es un tema de antigua data y cruza transversalmente a la sociedad. Actualmente, dadas las características del modelo económico imperante en Chile, resurge el tema del lazo social como prioritario en el análisis social. Al respecto, podríamos afirmar que el lazo social está en crisis o en proceso de cambio.

Éste ha sido el sujeto central de la sociología (Nisbet, 1970). Sin embargo, al estudiar la manera como los seres humanos viven en sociedad, las teorías del lazo social nunca han sido cuestionadas. Entonces, ¿por qué es necesario hacerlo en el Chile actual y en particular relacionada con los actos de violencia al interior de los establecimientos escolares?.

Las concepciones clásicas del lazo social, filosóficas o sociológicas, nacieron en contextos bien particulares, que tuvieron en común la desestabilización de la sociedades tradicionales: los filósofos del siglo de las luces (XVIII) 
emergieron de la degradación del universo medieval, del crecimiento y poder de la burguesía comerciante y del capitalismo, de las divisiones de la cristiandad y de la aparición de la ciencia moderna.

En esa época, donde el desarrollo de los conocimientos reforzaban la racionalidad, el método y el progreso, la razón devino un ideal que fue concretado por la revolución francesa, verdadero mito fundador de la sociedad liberal contractual.

En nuestro caso, el fenómeno que se vive en el país es el desarrollo de un proceso de modernización, unido a un proceso más largo e imperceptible a simple vista, cual sería un proceso lento de mutación cultural, que se verifica en la vida cotidiana de los jóvenes, sea cual sea el espacio sociocultural que vivan en su cotidianeidad. Es en ese núcleo de la vida juvenil donde se están verificando los cambios que interesa conocer.

Dicho de otro modo, ¿cómo se produce el cambio cultural "por abajo" o "por dentro" ${ }^{9}$ ? ¿En los actos cotidianos, en el trabajo, en el consumo, en las relaciones de pareja, en los proyectos personales, en el uso del tiempo libre, en los grupos de amigos, en el Colegio, el Liceo o en la Universidad?, etc.

Dado que es en ese nivel donde los jóvenes hacen su vida, ahí se debe escudriñar para comprender sus conductas. Son esas manifestaciones, muchas veces imperceptibles, las que van generando los grandes cambios epocales.

9 El concepto pertenece a Michel Molitor. Al respecto ver: Jeunes Travailleurs en situation précaire et Modèle Culturel de travail. En: Contribution à la Sociologie du Sujet. Sous la direction de Guy Bajoit et Emmanuel Belin. L'Harmattan. Collection Logiques Sociales. París. 1997 